\title{
PC operating system for measuring discrete biological responses during classical conditioning
}

\author{
JONATHON E. GIFTAKIS and ROBERT W. TAIT \\ University of Manitoba, Winnipeg, Manitoba, Canada
}

\begin{abstract}
A software package, written in C source code for MS-DOS 5.0 or higher IBM-compatible computers, was developed for the classical conditioning of discrete biological responses. The program's primary functions include collecting and storing conditioning parameters, controlling presentation of stimuli, transforming discrete analog responses into dependent measures (e.g., CR frequencies and various measures of response topography), and generating data sets for use with commercial PC statistical packages. These functions were implemented in a windows-based user interface to increase the experimenter's ease of use and to maximize efficiency in performing experimental procedures. Scrollable windows provide detailed visual displays that permit monitoring responses during conditioning and a review of each trial after the session is over. Additional software tools aid the visual inspection of response topographies, data manipulation, and calibration of the experimental apparatus. An overview of the system, its design objectives, and the user interface is presented.
\end{abstract}

Classical conditioning (Gormezano, 1966; Gormezano \& Kehoe, 1975; Patterson \& Romano, 1987) is a behavioral paradigm involving the presentation of two stimuli. One of these stimuli, designated the unconditioned stimulus (US), is biologically significant to the organism and reliably produces a measurable biological response labeled the unconditioned response (UR). The second, designated the conditioned stimulus (CS), does not initially elicit the biological response. By repeatedly presenting the CS and US in a specified temporal arrangement, the target response develops to the CS. The latter target behavior is denoted the conditioned response (CR) and is considered an index of associative learning.

Most classical conditioning experiments can be conceptualized as involving one or more phases, each of which contains multiple sessions in which a fixed sequence of stimulus events is administered to a group of subjects. A schematic representation of a typical conditioning session is displayed in the upper part of Figure 1. Throughout the session, CS-US pairings and consequent responses occur on temporally separated trials. Each trial is nested within an observation interval or window. Separating each observation window is an intertrial interval (ITI), either fixed or variable in duration and usually measured in seconds.

Events occurring within the much shorter observation window are depicted in the lower part of Figure 1. It is during the observation interval that stimuli are presented

This research was supported by Grant A0312 from the Natural Sciences and Engineering Research Council of Canada to R.W. Tait. J.E. Giftakis was supported by both an undergraduate and a postgraduate scholarship from the Natural Sciences and Engineering Research Council of Canada. Requests for reprints should be addressed to R.W. Tait, Psychology Department, University of Manitoba, Winnipeg, MB, Canada R3T 2N2 (e-mail: tait@bldgduff.lanl.umanitoba.ca). to the subject independent of its behavior. The temporal relationship among the stimuli is determined by controlling the onset and offset of each stimulus within the interval. On each trial, continuous recording is conducted throughout the duration of the observation interval, with a sampling rate ranging from 1 to $5 \mathrm{msec}$, to obtain an accurate representation of real-time analog responding. The observed response, which indicates that a change has occurred in the target system, is scored in reference to predefined scoring intervals (shown as dotted lines) tied to the temporal properties of the stimuli. Conditioned responses are indexed by onset latencies that occur within the scoring interval.

Thus, in conducting a classical conditioning experiment, the experimenter must be able to manipulate the temporal characteristics of the stimuli on any given trial (i.e., onset latency, duration, type, and number), control the time interval between successive trials, record the target response during a specified observation interval, and transform the collected data into dependent measures that operationalize the target response. These tasks have proven to be amenable to computer control.

Applications of computer technology to classical conditioning procedures have been implemented on various systems: DEC 4K PDP-8/E (Millenson, Kehoe, Tait, \& Gormezano, 1973; Tait \& Gormezano, 1974); Apple II/FIRST system (Scandrett \& Gormezano, 1980); and, more recently, IBM-compatible computers (Penttonen, Salmi, Hamalainen, \& Meriluoto, 1994). The present software package, written in C source code and implemented on MS-DOS 5.0 or higher IBM-compatible computers, was designed to expand the capabilities of earlier systems. The program implements response-independent stimulus control and analog-to-digital (A/D) recording capabilities appropriate for phasic response systems (i.e., nictitating membrane extension, jaw movement, head 


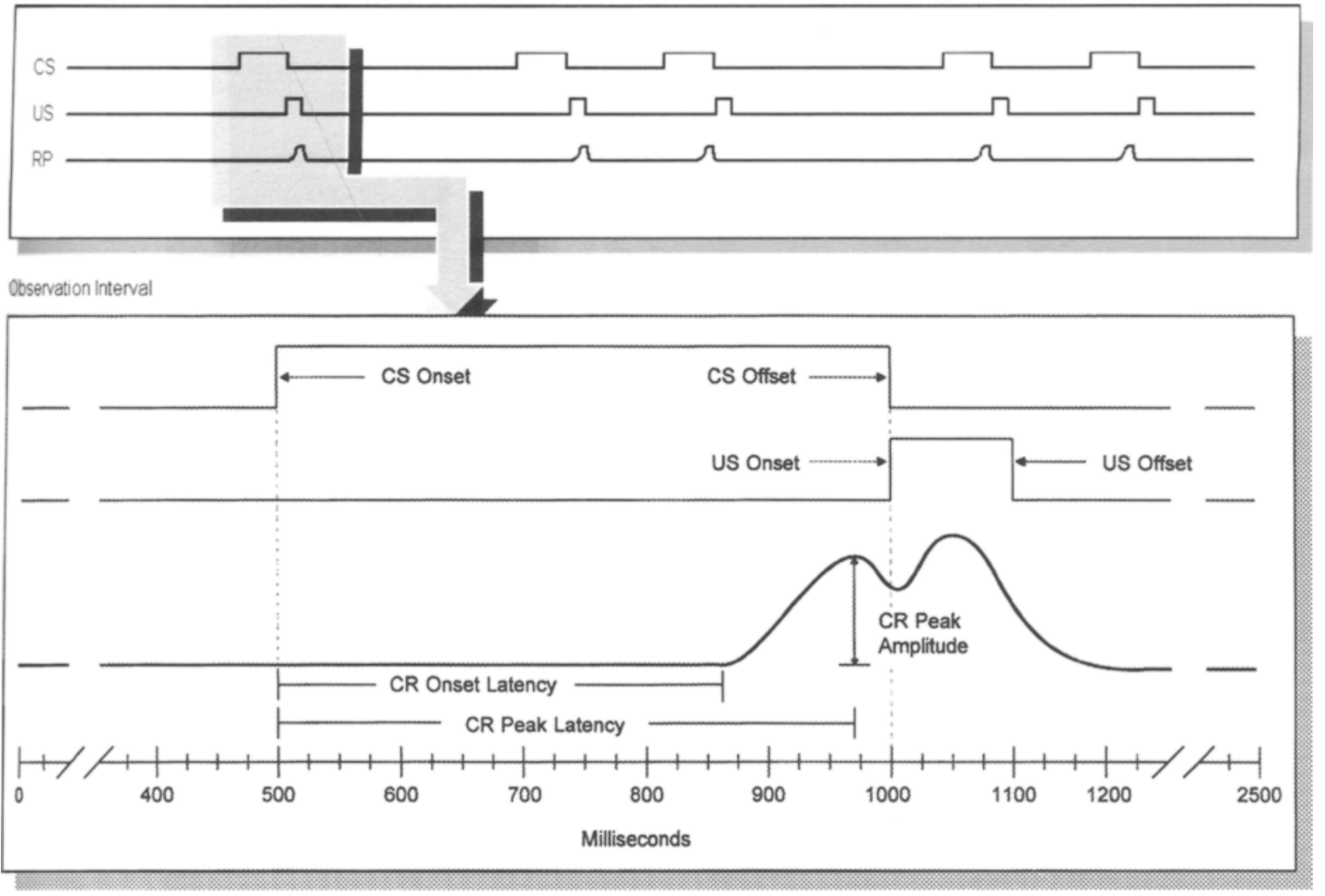

Figure 1. The upper part of the figure is a schematic representation of a typical conditioning session. CS-US pairings and consequent responses (RP) occur on temporally separated conditioning trials. The temporal separation between trials is called the intertrial interval. On-trial events occur during the observation interval depicted in the lower part of the figure. The response shown is characterized by a waveform that represents changes in the target system. This target behavior is labeled the conditioned response (CR) because it is under the stimulus control of the CS.

turning, etc.). Timing operations and data acquisition are performed by a National Instruments interface board using three independent 16-bit timers and a 12-bit A/D converter. The hardware allows for on-trial response recording to be performed using a 1 -msec sampling resolution for up to 16 subjects. A dual-monitor system displays numerical data (response amplitudes and latencies) and graphically illustrates the response waveforms between trials, which are marked for response-onset latency. Individual waveforms can also be amplified with pan and zoom features using scrollable windows. Postsession analyses include visual inspection of all responses collected, CR-frequency calculations, and measures of various waveform characteristics. All dependent variables are outputted in a spreadsheet format that is compatible with several commercial PC statistical packages. A more detailed description of the system and software architecture follows.

\section{SYSTEM OVERVIEW}

A representation of our microcomputer laboratory system for performing classical conditioning is displayed in Figure 2. An IBM microcomputer is connected to eight conditioning chambers (one is shown) situated in a sound-attenuated adjoining room. Within each chamber is a stimulus panel positioned directly in front of the subject. The delivery of stimuli is controlled directly by the software program, which communicates with stimulus devices via digital control lines. The experimental apparatus is similar to that described by Gormezano (1966) for conditioning of the rabbit's nictitating membrane response. The subject is placed in a Plexiglas restraining box, with the head fixed in position by foam pinnae clamps. A potentiometer is then positioned on top of the subject's head, with the armature connected to the membrane situated near the canthus of the eye. Horizontal displacements of the membrane, which occur in response to an aversively perceived stimulus, produce rotary movements in the potentiometer. These movements are recorded by the computer and displayed as waveforms on the VGA graphics display monitor for each subject. Stereotaxic measurements have shown a movement of $0.5 \mathrm{~mm}$ in the response system to correspond to an average output of 13 digital units when a $1-\mathrm{msec}$ sampling resolution is used. Response-onset latencies and amplitudes are displayed in 

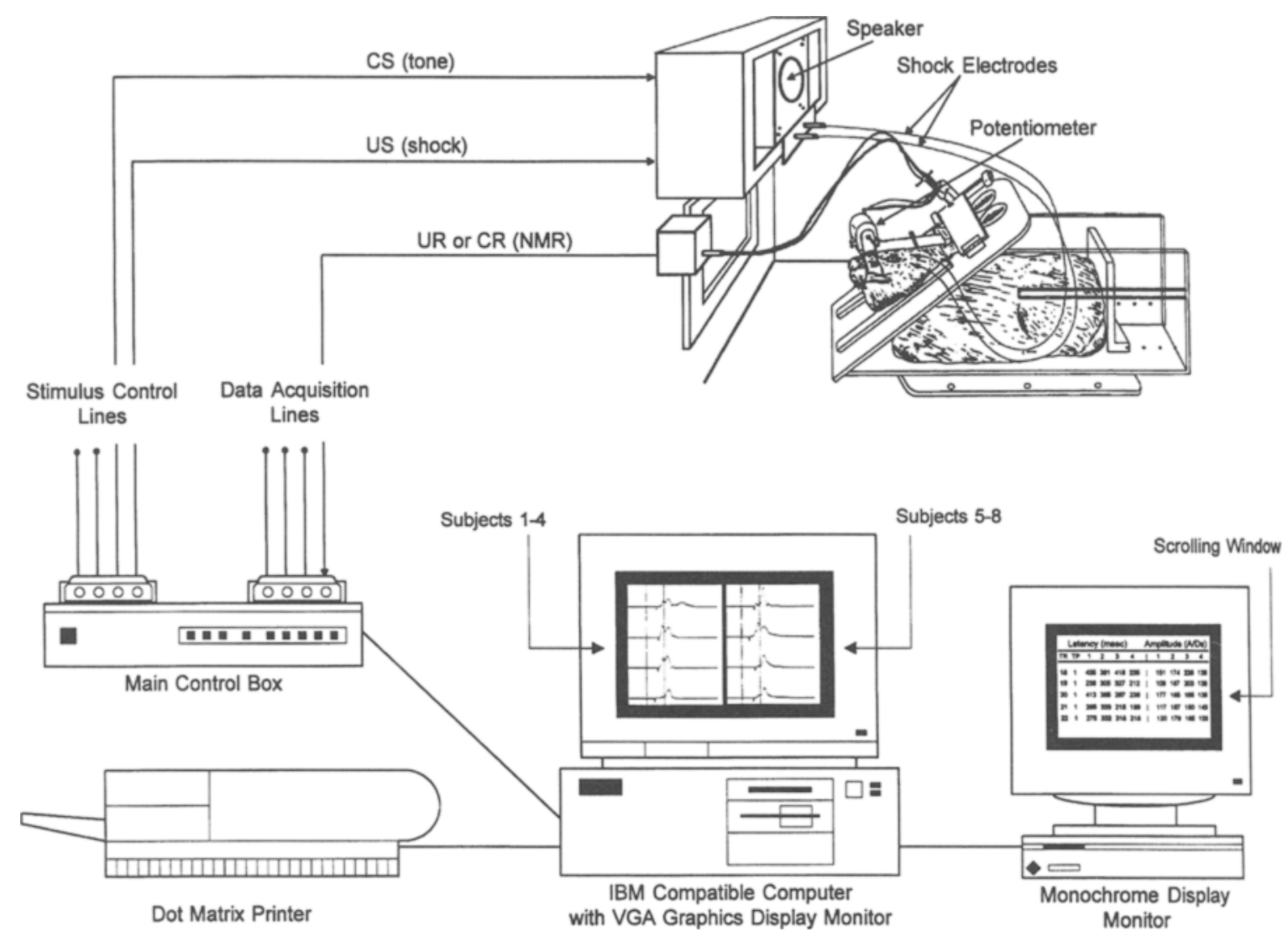

Figure 2. A representation of a microcomputer laboratory system for performing stimulus control and data acquisition during classical conditioning experiments. The experimental apparatus is similar to that described by Gormezano (1966) for conditioning of the rabbit's nictitating membrane response (NMR).

a scrollable window on the adjacent monochrome display monitor and printed following each trial. All experimental parameters and responses are automatically stored on either the hard drive (default condition) or to floppy disk.

\section{SOFTWARE DESIGN OBJECTIVES}

Four major design objectives focused the creation of the present system. The first objective was to make the operation of the package as easy as possible for the experimenter-that is, to make it "user friendly." This was accomplished by developing a windows-based menu interface for the collection of parameters and conditioning of subjects. With other software implementations, experimental parameters are created by writing program control language that must be compiled prior to each session (Penttonen et al., 1994; Scandrett \& Gormezano, 1980). This requires knowledge of programming that is both time consuming and difficult for novice users. The benefits of a windows-based interface are fewer errors and greater speed in initializing experimental sessions.

The second design objective was to provide detailed visual displays for the monitoring of responses during conditioning and for playback after conditioning. This was accomplished by using a two-monitor system, one to display numerical data for the most recent trials and one to display the waveforms collected on the immediately preceding trial. In addition, any waveform can be selected and amplified through pan and zoom features, which permit closer visual inspection with a variety of tools. Because the software stores the original data for each trial, the experimenter is also provided with the option of viewing subject responses after the experiment is completed. These options are unique to the present software program.

The third objective was to provide dependent-variable calculations and summaries that could be easily outputted to commercial statistical packages (i.e., SPSS or SAS). We achieved this objective by developing a transformation program that extracted selectable dependent variables from the response waveforms and saved them to file for analysis.

The last objective was to provide the experimenter with tools for calibrating equipment involved in response recording and stimulus control. With this aim in mind, the software was written to perform various calibration functions, such as controlling stimulus devices, identifying malfunctioning recording equipment, and defining criteria to use for response thresholds. 


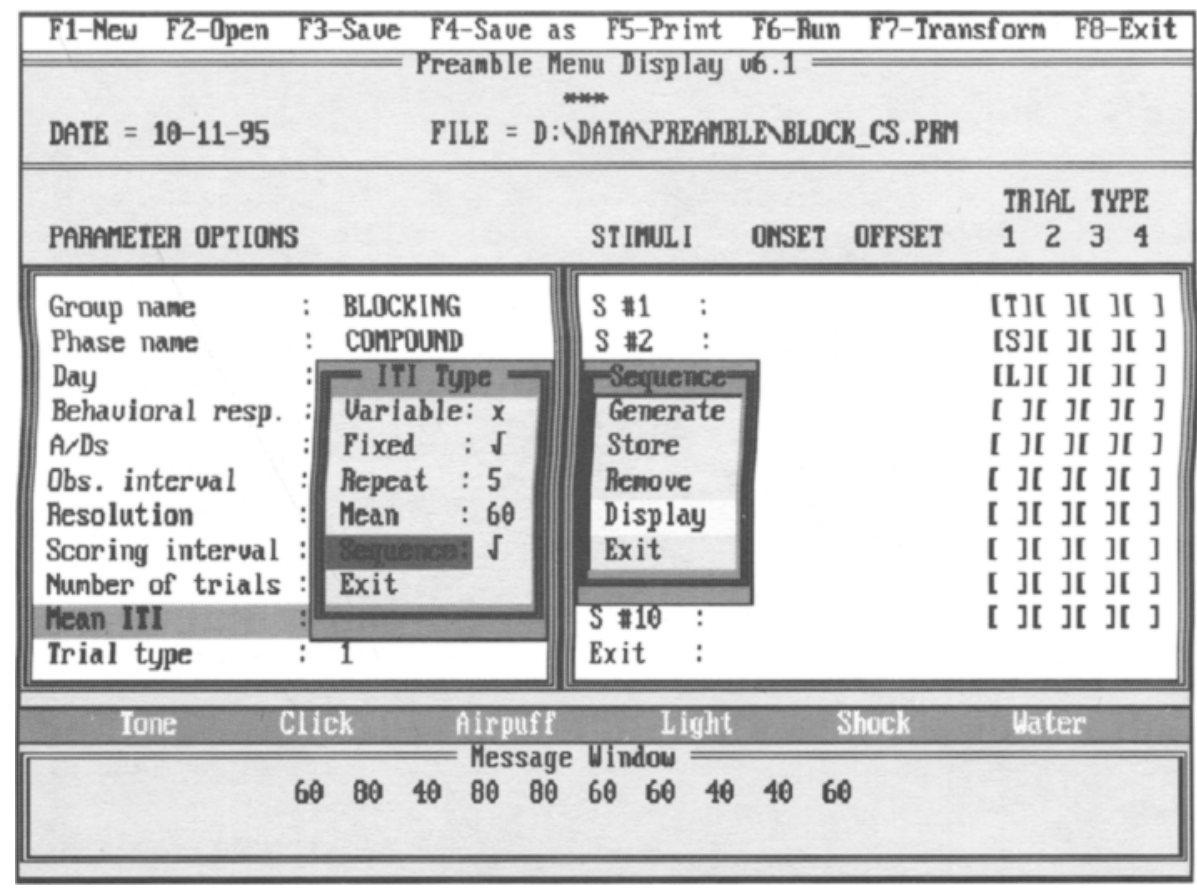

Figure 3. A view of the preamble window interface. The program is used to collect and store information required to define experimental procedures operationally.

\section{SOFTWARE ARCHITECTURE}

The main program may be conceptualized as consisting of three integrated components: the preamble, which collects and stores variables necessary for conducting the experiment; the conditioning program, which uses information from the preamble to control stimulus presentation and recording of responses; and the transformation program, which extracts dependent variable measures from the analog waveforms and outputs them to a data set. A separate installation program installs and configures the main program for the user. The program's integrative appearance is achieved by implementing a consistent interface throughout the different components. At the top of each screen is a main pull-down menu which can be activated by using either the keyboard or a mouse. The menu contains component-specific functions. Submenus are triggered for the specification of complex variables that require multiple values (i.e., multiple observation intervals), sequence processing (i.e., variable ITI values), and stimulus definition. At the bottom of each screen is a message window used to signal the user about menu options, inputting violations, and system errors. Each of the different components and the functions associated with them are discussed in the following sections.

\section{PREAMBLE}

The first step in conducting a conditioning session is to establish the parameters that operationally define the experimental procedures for each group in the study. To accomplish this task, the experimenter uses the preamble to collect and store the needed information. Figure 3 illustrates the preamble menu display with parameter information that has been entered for a typical conditioning session. Qualitative information, such as the name of the group and phase of the experiment, is collected as string inputs of up to 11 characters long and is used by the program to label printed output. The experimenter is able to control which behavioral response to record by selecting the appropriate response system. Although current options include the nictitating-membrane and jawmovement responses, or their combination, minor modifications can be made to the program code to measure other forms of analog responding (i.e., eyeblink, leg flection, head turning, etc.). The number of $A / D$ s defines the total number of individual responses to be simultaneously recorded for a single group of subjects, and can range from 1 to 16 , depending on the group size. This information is used by the program to determine which channels are to be sampled during a conditioning session. The experimenter also has the option of defining the sampling rate to be used for all channels (millisecond units ranging from 1 to 10 ).

\section{Intertrial and Observation Intervals}

The preamble provides the experimenter with a flexible means to operationalize trial sequences and stimulus events necessary for stimulus control. The logic underlying both processes is depicted in Figure 4. A trial sequence, which requires defining the ITI, is constructed in three steps. First, the number of trials and the mean time interval between successive trials (in seconds) is defined. Second, a repeat value is used to represent the num- 
Intertrial Interval Parameters

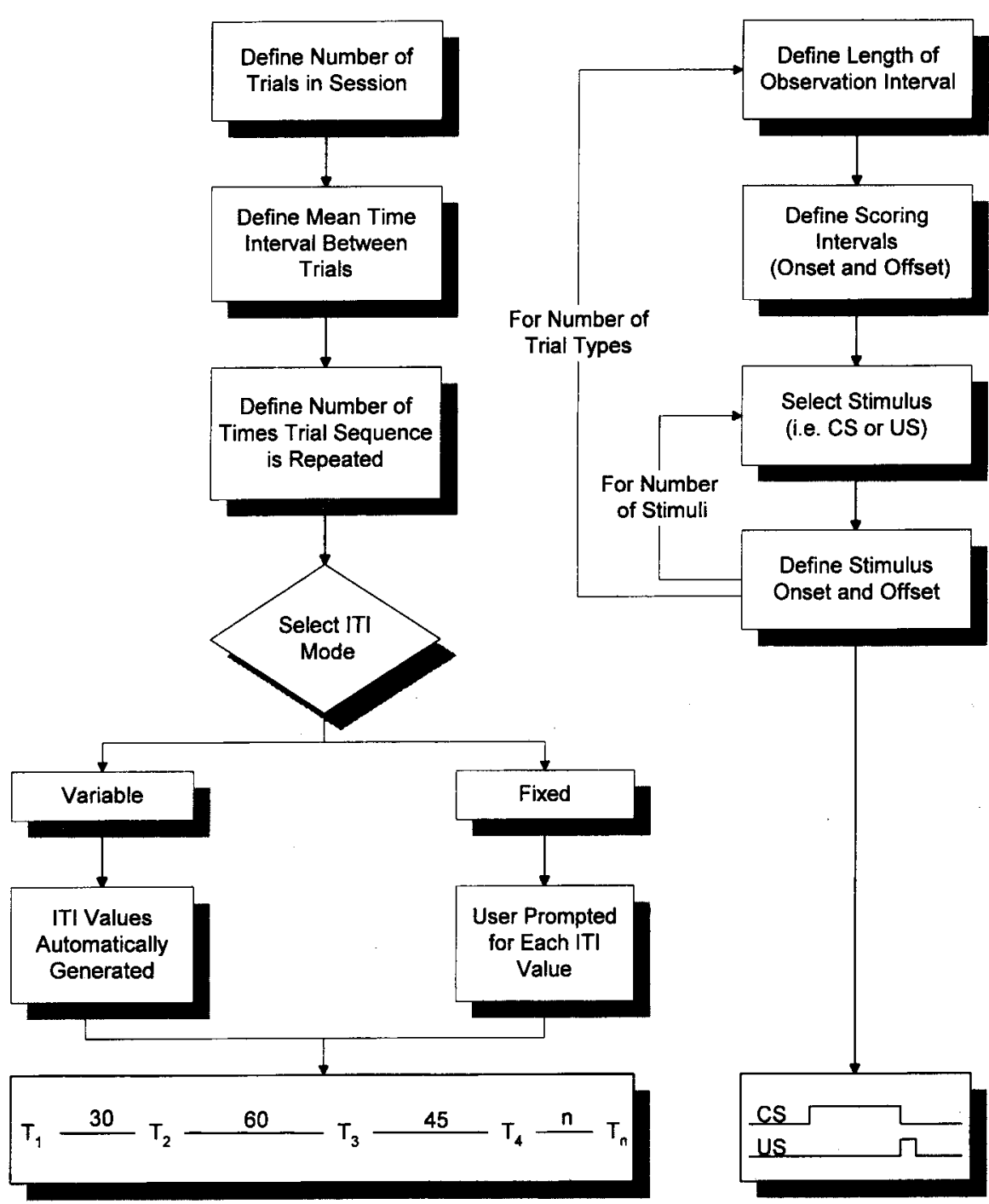

Figure 4. Logic used to establish trial sequences and stimulus events necessary for stimulus control.

ber of times the trial sequence is administered in the session. From this information, the program computes the number of required intervals. The third step requires selecting the mode for generating the ITI values: variable or fixed duration. When the variable option is selected, the ITI values are "automatically" generated, with the resulting sequence displayed in the message window (see Figure 3). Three unique interval times are created: one equaling the mean, a lower bound that is $2 / 3$ the value of the mean, and an upper bound that is $4 / 3$ the value of the mean. The interval times are randomly distributed, except that no more than two identical times can occur consecutively. In contrast, if the fixed option is selected, the user is prompted to enter a time value for each ITI in the sequence. A warning message is displayed if the interval times do not approximate the mean as the measure of central tendency. Once the ITI values have been generated, the experimenter is given the option of either storing or regenerating the sequence. Changes to a previously stored sequence are also possible.

Timing of stimulus events during a conditioning trial is achieved by defining observation interval parameters. Up to four observation intervals can be constructed for a session so that four different combinations of stimuli (trial types) can be used. The sequence of trial types is manipulated by selecting variable or fixed modes, depending on whether or not a random trial sequence is desired. Within each observation window is nested a scoring interval that is used to identify the occurrence of a CR. Typically, the scoring interval corresponds to the CS-US interval. The user specifies the duration of the scoring interval by defining its onset and offset relative to the beginning of the 


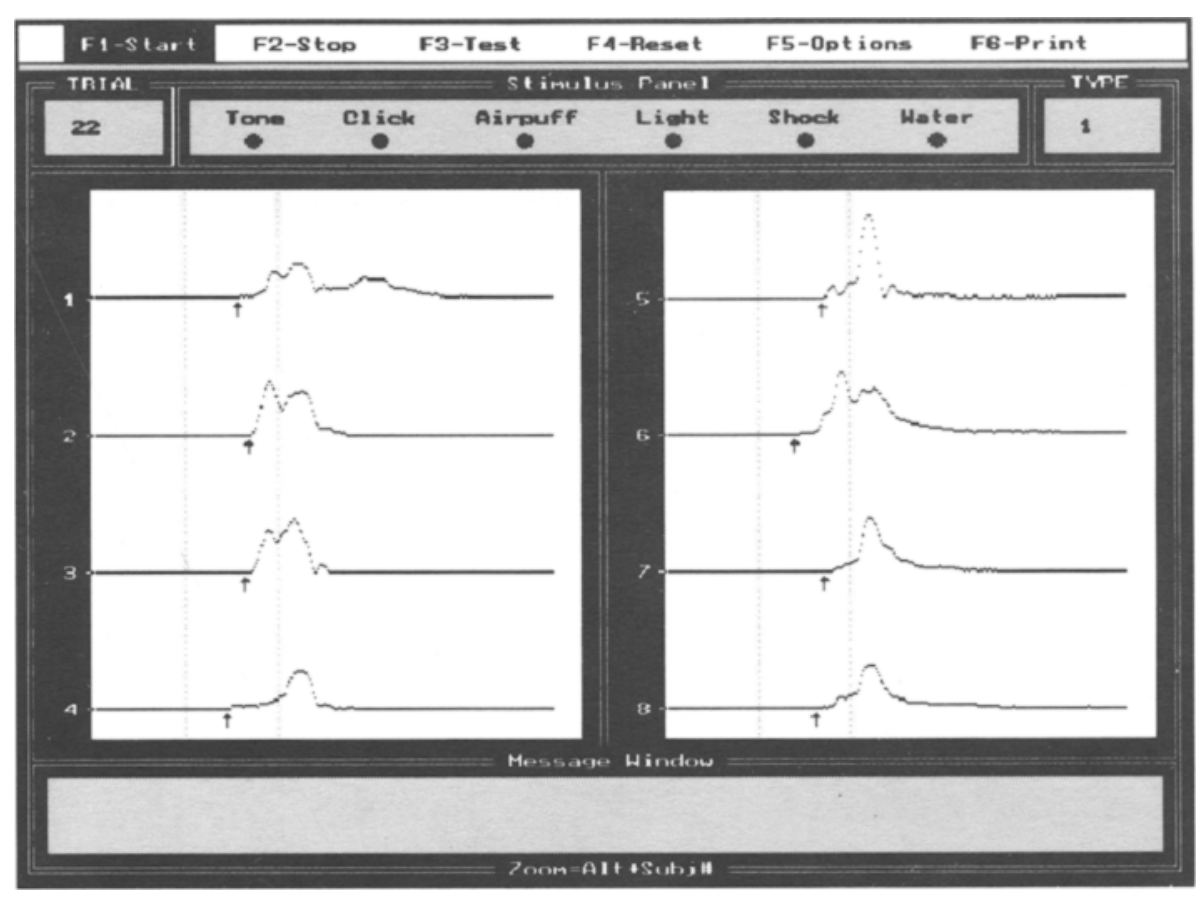

Figure 5. A view of the conditioning window illustrating the response waveforms for 8 subjects displayed during the intertrial interval. The dotted lines represent the onset and offset of the scoring interval. The onset latencies of the responses are marked by arrows.

observation window. To ensure that the conditioning program can establish a pre-CS baseline, the preamble restricts the onset of the scoring interval to a minimum of $500 \mathrm{msec}$. If the experimenter attempts to define a scoring interval that exceeds the length of the observation window, the observation-window dialog box is automatically called, allowing for a redefinition of its length.

Once the scoring intervals have been defined, the experimenter can select and define the stimuli to be used during each observation interval. To accomplish this, a stimulus panel is highlighted above the message window, displaying the labels for all possible stimuli that can be used in the experiment (e.g., tone, click, airpuff, light, shock, and water in Figure 3). Once a stimulus has been selected, the experimenter is prompted to define its onset and offset relative to the beginning of the observation window. The preamble checks to ensure that stimuli of the same type do not overlap with one another, and that their onset and offset values conform to the proper order and boundaries.

\section{Managing Experimental Procedures}

With most classical conditioning procedures, several sessions of training are required before a subject meets an acceptable behavioral criterion. As a result, multiple sessions are often administered using identical experimental procedures. For example, generating an acquisition curve requires repeated CS-US pairings administered over several consecutive days. It therefore follows that, in an efficient system, the experimental procedures would be defined only once but could be invoked again for additional training. Although this can be made possible by storing the parameters in a single file, a problem arises in that the output produced for each session of training is unique, and therefore must be stored separately under different file names. Our preamble circumvents this problem by using a file-storage system that can be indexed using the session number for any particular day of the experiment.

For example, the user may save the training parameters for a control group using the name "control." The file-storage system automatically appends the extension ".prm" to denote that the file contains preamble parameters ("control.prm"). The conditioning program then uses a modified form of the preamble file name to store the $A / D$ data. A unique file name for each day of training is automatically created by appending a designator representing the current session number and file type to the file title. During additional training sessions, the day field in the preamble is incremented by the experimenter, which automatically causes the file name used for data output to change ("control.01d" for Day 1, "control.02d" for Day 2, etc.).

To aid in managing the experimental procedures, the preamble allows the investigator to open, save, rename, and print each group's parameter file. When the user attempts to open a file, a scrollable dialog box appears listing all of the preamble files in the current directory. The number of free bytes available for storage is also indicated. A directory path-change feature allows the user to open files from any directory location or disk drive on 


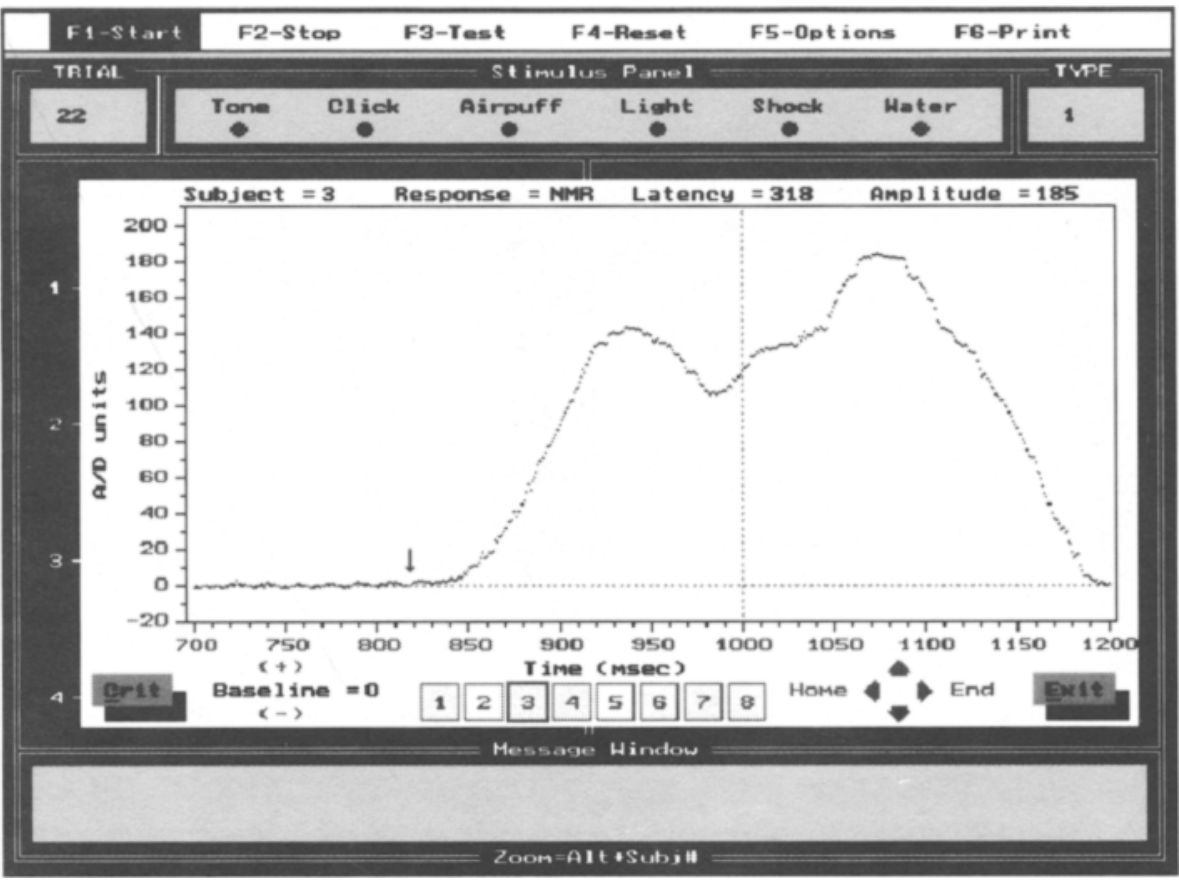

Figure 6. A magnified view of a conditioned response obtained in zoom mode. The response waveform is graphically displayed in its original 1-msec resolution using dynamically scaled axes and onset indicators. Options in this mode include subject switching, baseline fitting, response panning, and the ability to overlay threshold indices on the response.

the system. However, only files with a ".prm" extension are displayed and can be opened.

Choosing to save and rename files produces a similar dialog box, with the exception that the user is automatically prompted for a file name. Changes to previously stored parameters are permitted using an existing file name, although a message will warn the user before overwriting the file. The current parameters in the preamble can be cleared at any time, allowing for a new set of parameters to be defined. Because the experimental procedures are usually known in advance, we find it most efficient to define and save the parameters for all groups at the beginning of the experiment. Thus, when conducting the experiment, the experimenter needs only to increment the current session number at the beginning of each day of training to set up each session.

Before a training session is conducted, the preamble parameters can be printed, thereby documenting the experimental procedures that were used. We find it particularly useful to keep a copy of the experimental parameters and data together in a group's file. The user can select one of three resolutions for the printed output (low, medium, and high), which can be directed to one of two parallel ports for printing on a dot matrix printer.

\section{CONDITIONING PROGRAM}

Once the parameters have been established and saved to file, the experimenter can begin a conditioning session by selecting the "Run" option from the main menu. Fol- lowing a series of parameter checks, the preamble variables are passed to the conditioning program, where they are used to control classical conditioning procedures.

Figure 5 displays the waveform graphical display of the conditioning program. It is divided into three primary components: a stimulus panel that displays the presentation of stimuli to the experimenter in real time, an analog response area that displays the individual waveforms recorded for subjects during the observation interval, and status indicator areas for keeping track of the current trial, trial type, and the ITI count during the session.

\section{On-Trial Events}

The ITI clock, which is displayed in the upper right portion of the screen between trials, is controlled by selecting the "Start" and "Stop" options from the main menu. Once a session has been started, the clock counts down until the end of an ITI has been reached. At this point, the conditioning trial begins. The ITI clock disappears, the current trial type is displayed, and the stimuli are presented to the subjects while their analog responses are being recorded during the observation interval. Stimulus delivery occurs simultaneously in all conditioning chambers and is controlled using the same parameter set. As a means of monitoring stimulus control, each stimulus is depicted by a red indicator light that turns green during stimulus delivery. Response recording is accomplished by sampling the voltage output of a potentiometer fixed to each subject and storing the digitized values in a dynamically created memory buffer. The size of the 


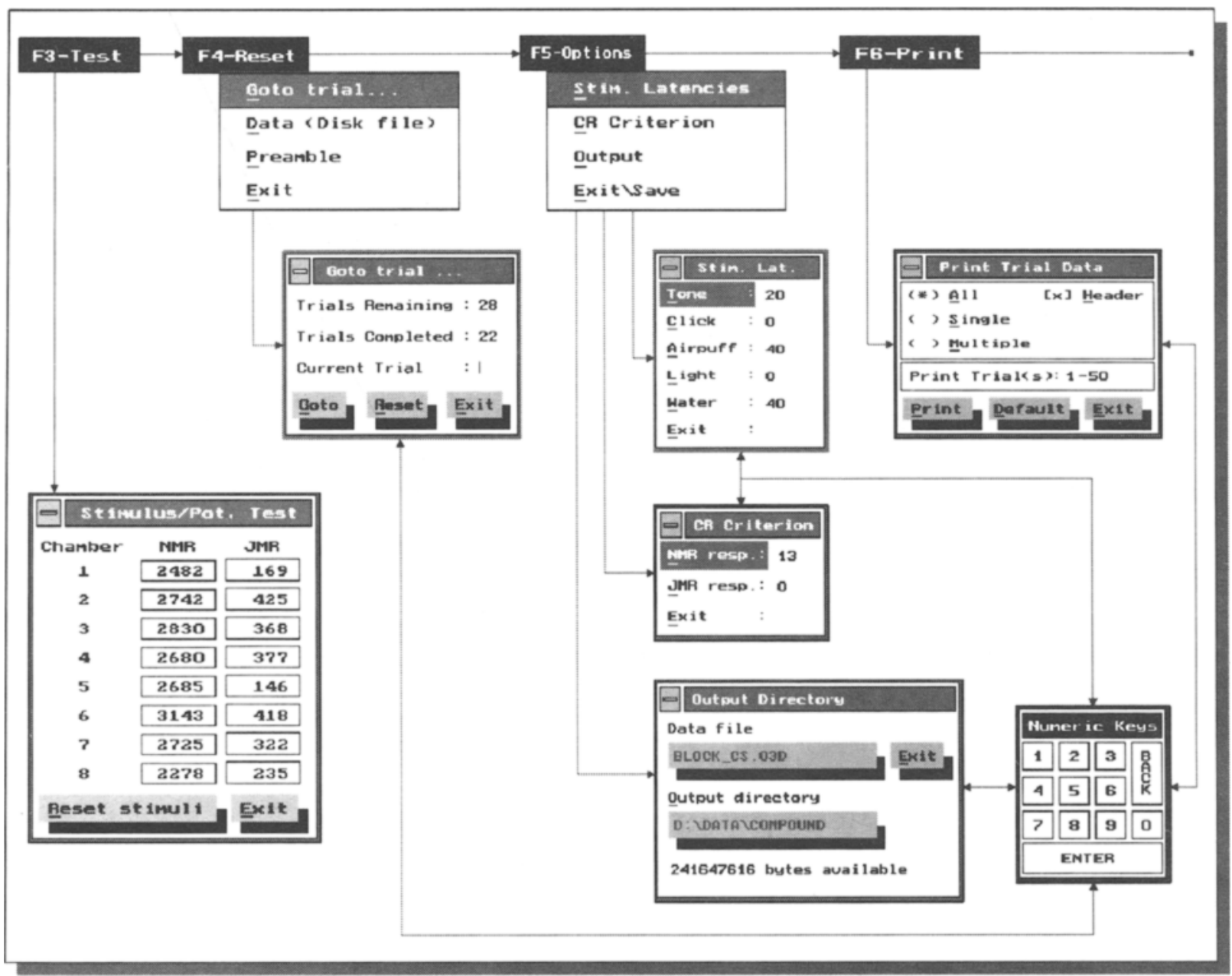

Figure 7. The menu structure of the conditioning program showing different options and functions. Program control is illustrated by the interconnections between options.

buffer is a function of the length of observation interval used, number of $A / D$ units, and sampling resolution. For instance, the maximum duration that 1 -msec samples can be recorded for eight $\mathrm{A} / \mathrm{D}$ channels is $2.5 \mathrm{sec}(20,000$ bytes). On each trial, the buffer is cleared and filled with new data that remains there for the duration of the intertrial interval, where it can be accessed by different functions of the program.

Following the trial, the $\mathrm{A} / \mathrm{D}$ data are automatically saved to a binary output file and the responses are scored for their onset latencies and peak amplitudes, which are then numerically displayed on a separate terminal (this is accomplished through coexisting VGA and monochrome video cards in the system). The response waveforms are graphically displayed on the VGA monitor, along with indicators to represent scoring intervals and onset latency measures (see Figure 5). The trial data are then printed and the next ITI is counted down. This sequence continues until the last trial of the session has been delivered.

The stop option can be used to suspend the system at anytime during the ITI. ITI countdowns and other eventscheduling parameters are preserved until the system is resumed. The interrupt feature was designed to allow the experimenter to attend to subjects during the experiment without disrupting on-trial events.

\section{Zoom Mode}

During the ITI, the responses for all subjects are simultaneously displayed on the graphics monitor. Each response is depicted using a plotting resolution of $10 \mathrm{msec}$, although the dependent measures are calculated using the higher resolution. However, the experimenter may select an individual response for a more detailed visual analysis. By selecting the channel number corresponding to a particular waveform, either by using the mouse or an ALT-number key combination, the response is magnified in a scrollable window. An example of a magnified response for Subject 3 is illustrated in Figure 6. The arrows at the bottom right of the zoom window allow the experimenter to pan the response in any direction, allowing for a full view of the response throughout the observation interval.

With the zoom mode, the experimenter is also provided the option of manipulating the baseline to provide 
a better fit to the data. In addition, the experimenter can also choose to superimpose a CR criterion line on the response to determine when it has been reached or exceeded. Both options aid the experimenter in accurately defining the characteristics necessary for proper waveform analysis.

A magnified view of a different subject's response is made possible by selecting one of the buttons located at the bottom center of the zoom window, each of which represents a different $A / D$ channel. When a new response channel is selected, its onset, identified by an arrow, is automatically positioned so that it occurs near the middle of the zoom window. Responses that significantly deviate from the baseline prior to the end of the scoring interval but do not reach CR criterion are marked with an arrow of a different color, and are not scored for analysis.

Because the operations of the zoom window are multitasked with the ITI clock, the ITI count is not disrupted when the previously described tasks are performed. However, $5 \mathrm{sec}$ prior to an upcoming trial, all pan and zoom operations are automatically terminated and the zoom window is removed from the desktop. This ensures that the system has sufficient time to initialize itself for processing events during the upcoming observation interval.

\section{Conditioning Parameters}

The preamble functions to collect and store the conditioning parameters that are unique to each experiment. However, the conditioning program also requires that some additional parameters be defined. Because these parameters are closely tied to the conditioning process and experimental apparatus, and therefore do not usually change between experiments, they are defined and stored by the conditioning program instead of the preamble. Figure 7 shows the different options and functions of the conditioning program, as well as the interconnections between them.

Stimulus latencies. Because stimuli have physical properties that affect their transmission rate, this factor must be taken into account so that the experimenter's temporal specifications are the values received by the subject. Otherwise, the subject may be receiving the stimulus at the wrong time during the trial. For example, if the experimenter defines the airpuff stimulus to begin $500 \mathrm{msec}$ relative to the onset of the observation interval, but it takes $40 \mathrm{msec}$ to reach the subject from its source, then the CS is actually received at $540 \mathrm{msec}$ and not at $500 \mathrm{msec}$. To correct for this difference, the conditioning program allows the experimenter to define a latency for each stimulus, which is automatically subtracted from the intended latency of that stimulus (i.e., the one defined in the preamble) every time it is in use. Thus, in the example, the airpuff stimulus would be activated at $460 \mathrm{msec}$ so that the subject received it at $500 \mathrm{msec}$ into the observation interval.

CR criterion. In addition to providing parameters for stimulus control, the conditioning program allows the user to establish how the response is to be defined. This is accomplished by specifying a CR criterion, which represents the amount of change from the A/D waveform's resting state that is equivalent to a predefined behavioral threshold of movement by the subjects. In our system, a separate CR criterion can be defined for each response system. When attempting to change the $\mathrm{CR}$ criterion from its existing value, a message is displayed showing the number of $A / D$ units that correspond to $0.5 \mathrm{~mm}$ of movement in the target system, a common criterion used in classical conditioning studies.

A/D data storage location. The output option in the main menu allows the experimenter to view the name given to the datafile that stores the binary output for each conditioning trial. Both the datafile name and the directory in which it is located are displayed. The file name is automatically created using conventions necessary for linking it to its corresponding preamble file and, as a cautionary measure, cannot be modified from within the program. In contrast, the output directory can be changed to direct the binary file to any path location or available drive on the storage device. We find this feature useful because it allows us to store the conditioning data for each phase of an experiment in separate directories. In addition, it allows the experimenter to change to a different path location either before or during an experiment if the reported number of available bytes becomes too low.

\section{Printing Trial Data}

Following each conditioning trial, the onset latencies and peak amplitudes of responses for all subjects are printed on the dot matrix printer (this is the same data that is displayed on the monochrome monitor). Thus, at the end of the session, the experimenter is provided with a hard copy of the data for all trials in the session. However, situations sometimes arise that prevent such records from being obtained; these include mechanical failure of the printer (i.e., it is off-line or out of paper) or errors made by the experimenter. In such cases, printing of trial data is suspended but the experiment continues. Because timing operations are not affected, printer problems can be corrected without disrupting the experiment. In addition, complete records can be created at the end of the session.

To obtain a hard copy of the data after the session, the print option can be selected from the main menu. It allows the experimenter to print the data for all trials or a selectable range of trials. In addition, there is also the option to print the header that appears at the top of the output page, which contains relevant information about the conditioning session (i.e., datafile name, algorithm version, response criterion, and indices for the trial, trial type, and subject). Before trial data is printed, the range of trials to print is displayed and the experimenter is prompted for confirmation prior to the starting of the printing process, which can be aborted at any time.

\section{Play-back Mode}

Anytime after the data have been collected, the experimenter has the option of "replaying" the experiment. The ability to play back an experimental session allows 


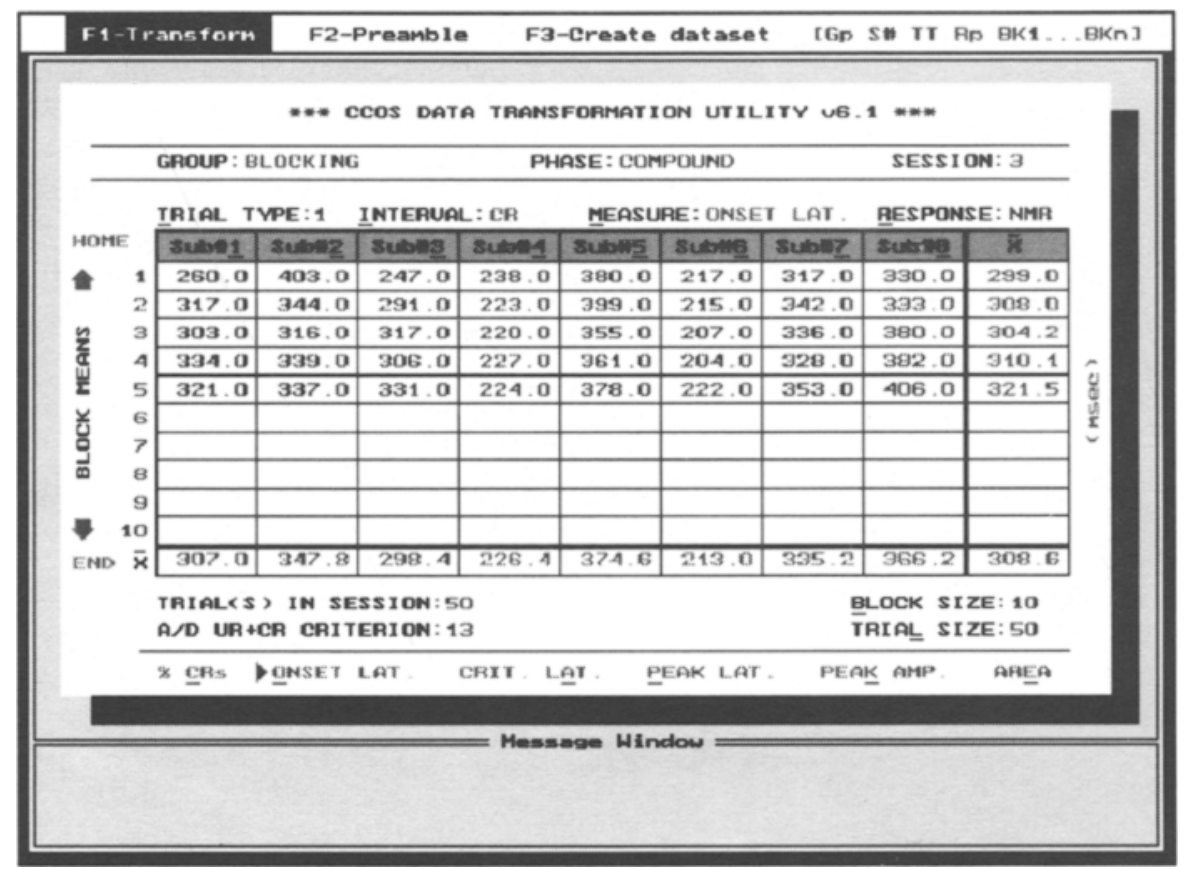

Figure 8. A view of the transformation window. The data in the matrix were obtained by reconstructing the response waveforms and extracting the dependent variables depicted below the matrix. Data manipulation options include block size, trial size, and subject selection. The program generates data sets in a file format compatible with commercial statistical packages.

for a detailed visual inspection of responding that may not be possible during the time frame of the actual conditioning session. This is performed by selecting the data option, which changes the mode of operation from normal to playback. In play-back mode, the user is able to view response topographies from all trials in the session, which are reconstructed by using the $A / D$ data values from the binary output file. The rate of playback is controlled directly by the experimenter through mouse or keyboard commands. In addition, the experimenter can advance to any trial in the session by using the "goto trial" option of the main menu. A reset option clears all trial information and points the user to the first trial in the session. The response zooming and printing features available in the standard operating mode are also available in play-back mode.

\section{Calibration Features}

In addition to controlling classical conditioning experiments, the conditioning program was designed to assist in calibrating the experimental apparatuses used for response recording and stimulus control. Calibrations of both features can be accomplished by selecting the test option of the main menu. This option gives a dynamic view of the voltage output for the channels located in each conditioning chamber, as well as the option for operating each stimulus separately. The digitally displayed voltage values are refreshed at $1-\mathrm{sec}$ intervals and used primarily to set voltage ranges on potentiometers, identify malfunctioning $\mathrm{A} / \mathrm{D}$ lines and potentiometers, and calibrate the $\mathrm{A} / \mathrm{D}$ lines to establish the $\mathrm{CR}$ criterion. A message informs the experimenter of the target range required for potentiometer calibration. The stimulus test, which is activated by choosing one or more stimuli from the stimulus panel, can be aborted at any time by deselecting the stimulus or using the reset stimuli option. Having the ability to directly control each stimulus in the system enables the experimenter to adjust the temporal and physical characteristics of each stimulus prior to and between sessions of an experiment.

\section{System Errors}

System errors may occur during a conditioning session due to (1) apparatus failure, most often due to displaced or malfunctioning potentiometers, (2) power shortages that result in system shutdown, and (3) problems with permanent storage of the A/D data caused by insufficient disk space. Although these problems cannot always be avoided, they can be controlled to minimize any disruptive effects that may be produced during an experimental session. For example, a displaced or malfunctioning potentiometer produces a voltage reading that is outside the calibrated target range. The conditioning program uses this information to report that a measurement error has occurred, which would otherwise appear as a "no response." The ability to differentiate errors from no responses is especially important in experiments that use nonreinforced trials, where a lack of responding may be used to infer information about an associative process. 
After each trial, the current location in the trial sequence is stored in a configuration file. In the event of a system crash, restarting the conditioning program will automatically advance the experimenter to the last previously completed trial (with no loss of data!). In addition, if the experimenter runs out of permanent storage space in the middle of a session, the program prompts the experimenter to change the location of the output directory. The new location is then used for saving trial data for the remainder of the session. The two data files can be combined after the session is over.

\section{TRANSFORMATION PROGRAM}

The transformation program is used after the session is over to extract the dependent variables from the ana$\log$ waveforms and output them to a data set for statistical analysis. The graphical interface of the program, which is depicted in Figure 8, consists of a two-dimensional scrollable matrix (subject $\times$ block). Data-manipulation features include specifying the range of trials to use for analysis, combining trials into blocks of various sizes, and subject selection. To transform the data, the experimenter must select the transform option from the main menu. During the transformation process, the response waveforms for each trial are reconstructed (internally) and analyzed to produce the topographical dependent measures labeled under the matrix (\%CRs, onset latency, criterion latency, peak latency, peak amplitude, and area). The topographical measures are parsed by trial type and computed for three different intervals: $\mathrm{CR}, \mathrm{UR}$, and $\mathrm{CR}+\mathrm{UR}$. This combination gives rise to a total of 16 possible dependent variables.

The results of the analysis are printed to file and entered into the cells of the matrix (\%CRs is the default start-up screen). Additional calculations are made to obtain the row (block) and column (subject) marginal means, which dynamically change with modifications to the block or trial size. Each measure is represented by a separate screen and can be viewed by selecting the appropriate combination of variable and interval from the interface. Subjects can be removed from the analysis by using the keyboard or mouse to select its corresponding column in the matrix. The column becomes "grayed out" for all screens, and the marginal calculations are recomputed to reflect the change. Once the data have been transformed, it can be viewed at a later time for further inspection.

One of the powerful features unique to this program is its ability to create data sets for any of the dependent measures. This both saves time and reduces the number of errors that commonly occur when creating large data sets. To use this option, the experimenter provides the program with two pieces of information: the file name to store the data and a code defining the group. The "create data set" option saves the contents of a current data screen to file along with the necessary codes required for computing descriptive and inferential statistics. The coding format is displayed in the upper right portion of the interface (i.e., each column contains group number, subject number, trial type, response code, Block 1, Block 2, $\ldots$, Block $n$ ). To save a different measure, the experimenter simply switches to the appropriate screen and provides the program with a new file name. Because the program automatically stores the file names for each measure, they need to be defined only once for a session. This allows data for additional groups in the session to be appended to the existing file with only a modification to the group code.

Although the program is capable of creating multiplegroup data sets, it was not designed to merge these data sets across sessions. The reason for this is that sophisticated merging utilities exist in commercial statistical packages (e.g., SPSS Version 6.1) that successfully work with our data formats. Accordingly, we use the commercial packages to form the matrices and perform the analyses.

\section{SYSTEM REQUIREMENTS AND AVAILABILITY}

The software we have designed was written in Borland Turbo C (version 2.0) source code (36,562 lines) and compiled into five executable files (installation program, title startup screen, preamble, conditioning program, and data transformation program). Timing operations, stimulus control, and data acquisition functions are performed using a National Instruments interface board (Model ATMIO-16F-5) and an appropriate laboratory system. Software control of these functions was made possible by linking the appropriate function call routines, provided by National Instruments NI-DAQ library (version 4.3), to the $\mathrm{C}$-memory model. A technical representative at $\mathrm{Na}$ tional Instruments has indicated that our application should be compatible with newer versions of the interface board (Model AT-MIO-16E-2). However, an update of the NI-DAQ software driver (NI-DAQ version 4.8 , the latest to include DOS support) is required. The 1996 cost of the board was $\$ 2,400$ (CAN).

The minimum system requirements needed to operate the classical conditioning system are as follows: 386 IBM-compatible computer $(33 \mathrm{MHz})$ with $1 \mathrm{MB}$ RAM (600K free conventional memory), $60-\mathrm{MB}$ free hard disk space, MS-DOS 5.0 or higher, VGA video card, color display monitor, and dot matrix printer. The second terminal screen is recommended but optional; it requires a monochrome video card and display monitor.

The software can be obtained by sending two 3.5 -in. high-density IBM-formatted diskettes, along with a request letter and a \$50 fee (CAN), to the authors. Interested researchers should note that some modification of the source code is likely required before the software can be used in other conditioning labs. For stimulus control and data acquisition, the software uses mapping routines that are fixed to the hardware. Therefore, changes to the source code are required if either different hardware or mappings are used. In future updates of the software, the intent is to make these mappings directly controllable by the user. 


\section{CONCLUSION}

We have been using the operating system for approximately 2 years. Recently completed experiments have proven it to be very stable. That is, there have been no system failures attributable to software problems. All design objectives have been met successfully. Novice users in our laboratory have little difficulty with the package. Because they now can focus on mastering the procedures rather than learning how to program the computer, their training time has been reduced. The ability to move data directly to statistical packages has reduced both the time to complete analyses and the frequency of transcription errors. In addition, the ability to save all analog-to-digital records has allowed us to revisit experiments and analyze topographical features that were not initially the primary dependent variables.

\section{REFERENCES}

Gormezano, I. (1966). Classical conditioning. In J. B. Sidowski (Ed.), Experimental methods and instrumentation in psychology (pp. 385420). New York: McGraw-Hill.
Gormezano, I., \& Kehoe, E. J. (1975). Classical conditioning: Some methodological-conceptual issues. In W. K. Estes (Ed.), Handbook of learning and cognitive processes: Vol. 2. Conditioning and behavior theory (pp. 143-179). Hillsdale, NJ: Erlbaum.

Millenson, J. R., Kehoe, E. J., Tait, R. W., \& Gormezano, I. (1973). A minicomputer program for control and data acquisition in classical conditioning. Behavior Research Methods \& Instrumentation, 5 , 212-217.

Patterson, M. M., \& Romano, A. G. (1987). The rabbit in Pavlovian conditioning. In I. Gormezano, W. F. Prokasy, \& R. F. Thompson (Eds.), Classical conditioning (3rd ed., pp. 1-36). Hillsdale, NJ: Erlbaum.

Penttonen, M., Salmi, M., Hamalainen, P., \& Meriluoto, J. (1994). A microcomputer system for controlling classical conditioning experiments. Behavior Research Methods, Instruments, \& Computers, 26, 447-453.

SCANDRETt, J., \& Gormezano, I. (1980). Microprocessor control and A/D data acquisition in classical conditioning. Behavior Research Methods \& Instrumentation, 12, 120-125.

TAIT, R. W., \& GoRmEZANo, I. (1974). A microcomputer program for stimulus control and analog data for discrete trials paradigms in biological preparation: Classical conditioning. Behavior Research Methods \& Instrumentation, 6, 295-300.

(Manuscript received May 8, 1996; revision accepted for publication October 11,1996$)$ 